\title{
Serum laminin, hydrocarbon exposure, and glomerular damage
}

\author{
P Hotz, N Thielemans, A Bernard, F Gutzwiller, R Lauwerys
}

\begin{abstract}
It has been postulated that occupational exposure to hydrocarbons may damage the kidney and lead to glomerulonephritis and chronic renal failure. As laminin is a ubiquitous basement membrane component that seems to play a central part in the structure and function of basement membranes and as the normal renal filtration process is highly dependent on an intact glomerular basement membrane, the serum laminin concentration was examined in a population of workers exposed to hydrocarbons. The hydrocarbon exposure was assessed by exposure surrogates (exposure duration and exposure score). An interaction between occupational exposure to hydrocarbons and hypertension increased the laminin concentration whereas the laminin concentration decreased in workers exposed for a long time probably because of a selection effect. In a subgroup of printers exposed to toluene whose hippuric acid excretion had been recorded for several years this interaction was confirmed when the hippuric acid excretion was substituted for the other exposure indices. In the exposed group, the agerelated decline in creatinine clearance was accelerated. These results seem to confirm that occupational exposure to hydrocarbons is a non-specific factor that may promote a deterioration of renal function.
\end{abstract}

(British Fournal of Industrial Medicine 1993;50:1104-1110)

Institut für Sozial- und Präventivmedizin, Sumatrastrasse 30, CH-8006 Zürich, Switzerland P Hotz, F Gutzwiller

Unité de toxicologie industrielle et de médecine du travail. Clos Chapelleraux-Champs 30, B-1200 Bruxelles, Belgique

N Thielemans, A Bernard, R Lauwerys
Laminin is a ubiquitous component of basement membranes that seems to play an important structural and functional part within basement membranes. Indeed, this glycoprotein possesses binding domains for heparan sulphate proteoglycan and nidogen and cell binding properties involved in the anchorage of epithelial and endothelial cells to the glomerular basement membrane. Polyanionic charges of heparan sulphate proteoglycan are probably implicated in the ionic control of filtration through the glomerular basement membrane and nidogen mediates the binding of laminin to collagen IV, another major component of basement membranes. ${ }^{1}$ As the normal renal filtration process is highly dependent on an intact glomerular basement membrane it is not surprising that the serum laminin concentration may increase ${ }^{2}$ and that antilaminin antibodies may appear in patients diagnosed with renal disease. ${ }^{3}$

As organic solvents are suspected of playing a part in chronic nephropathies, Viau $e t a l^{4}$ searched for antilaminin antibodies in refinery workers exposed to hydrocarbons and found a slightly increased prevalence of high titres in exposed workers. Furthermore, Mutti et $a \bar{l}$ have recently described an increased serum laminin concentration in workers exposed to perchloroethylene. In both studies however, workers diagnosed with a renal or systemic disease were excluded. This might cause a selection bias as the workers with a history of kidney disease might constitute the group most at risk. Indeed, if renal disease is worsened by occupational exposure to hydrocarbon, the renal disease will progress more rapidly and it will be discovered earlier. Therefore, these workers will be excluded although hydrocarbons may have played a part in the progression of their renal disease. As a selection process of this kind is thought to be an explanation for the paradoxical association between albuminuria and ethnicity found in the cross sectional study of Gerber et $a l,{ }^{6}$ the possibility that studies including only healthy workers have underestimated the real risk of hydrocarbon nephrotoxicity in occupationally exposed workers cannot be excluded. 
The purpose of this study was to measure the serum laminin concentration in a population of workers exposed to hydrocarbons, including subjects diagnosed with previous or present renal disease or hypertension or both. The hypothesis was that a disturbance in the glomerular basement membrane is induced by the interaction between hypertension and occupational exposure to hydrocarbons and, therefore, that serum laminin concentration would increase especially in the exposed and hypertensive workers. This hypothesis is based on the results of a previous study with other markers of renal damage. ${ }^{78} \mathrm{We}$ also attempted to confirm these results with a biological indicator of exposure rather than indirect exposure indices such as exposure duration and exposure score. Therefore, a group of toluene exposed printers whose hippuric acid excretion was known was examined. Finally, the relations between serum laminin or heparan sulphate proteoglycan (assessed as total urinary glycosaminoglycan excretion) and other markers of glomerular damage were examined.

\section{Subjects and methods}

The study population involved adult male workers who were either invited to a general health examination in the frame of studies assessing occupational risks or had to undergo a periodic or preplacement medical examination. To avoid a recall bias, patients referred to us because of a possible occupational disease were excluded.

A detailed description of the selection rationale, of the products used (mainly toluene alone or in combination), of the characteristics of the subgroups, and of the study protocol has been reported elsewhere. ${ }^{79} \mathrm{Six}$ subgroups were identified-namely, floor layers (heavy hydrocarbon exposure; $n=106$ ), printers (moderate exposure; $n$ $=25$ ), roadmen (slight exposure; $n=40$ ), controls (no exposure at all, $n=48$ ), formerly exposed workers (former exposure only; $n=35$ ), "false" controls (present exposure not suggested by the present job title; $n=10)$. Fifteen $(5 \cdot 4 \%)$ workers did not participate (10 refusals, five because of organisational problems). There was not enough serum for laminin analysis in 20 cases and five workers had no satisfactory urine collection (protocol not observed, too little urine).

A hydrocarbon exposure is defined as an exposure resulting from any of 27 occupations listed in a standardised questionnaire. The exposure indicators are the same as in our previous work. ${ }^{78}$ Briefly, lifetime exposure duration (LED, years) and lifetime exposure score (LES; arbitrary units, years $x$ intensity factor) were assessed on the basis of the occupational history. Three exposure duration subgroups were used (ED1, no hydrocarbon exposure at all: $\mathrm{ED} 2$, between 0.01 and 9.9 years, ED3; $\geqslant 10$ years). Work related hydrocarbon induced symptoms (feeling drunk, having a non-specific gastric disturbance, dizziness, nausea, alcohol intolerance, or drowsiness) occurring during the four to eight weeks before the clinical examination were considered as a crude index of recent excessive exposure to hydrocarbons. A symptom was defined as work related if there was a clear work related pattern and when this relation was considered as probable. Doubtful or possible relations were therefore classified as non-work related. Workers complaining of at least one such work related symptom were compared with workers without any such symptom. Workers complaining of symptoms of this kind that could not be considered as work related constituted a third subgroup.

In the printer group, the exposure could also be estimated on the basis of hippuric acid (HA) concentrations in urine. As the individual HA concentration had generally been measured every three months since 1978 , individual yearly means of HA excretion could be calculated. Their sum for each worker gives the individual cumulative HA excretion. Details about these surveys and the analytical methods can be found elsewhere. ${ }^{10}$ The examined printers represent the whole present workforce of a printing house where lead was never used.

Exposure to lead, mercury, or cadmium was looked for with a check list. If possible, an intensity score was also assessed blindly by an industrial hygienist. $^{7}$

Serum laminin $(\mathrm{U} / \mathrm{ml})$ was assayed by measuring its pepsin resistant fragment (laminin P1; Behringwerke, Marburg, Germany). Creatinine in blood and urine was assayed by the Jaffé method (Boehringer Diagnostica, Mannheim, Germany) and the endogeneous creatinine clearance was measured over some hours $\left(\mathrm{ml} / \mathrm{min} / 1.73 \mathrm{~m}^{2}\right.$; median sampling time 187 minutes). Albumin excretion rate (U-alb; $\mu \mathrm{g} / \mathrm{min}$ ) was assayed by radioimmunoassay (Pharmacia, Uppsala, Sweden). The fractional albumin clearance was calculated as (urinary albumin/serum albumin) $\times$ (plasma creatinine/urinary creatinine). $\mathrm{N}$-acetyl- $\beta$-Dglucosaminidase activity (NAG; $\mathrm{mU} / \mathrm{mmol}$ creatinine), glycosaminoglycan concentration (GAG), erythrocyte counts (number $/ \mathrm{ml}$ ), retinol binding protein (RBP; $\mu \mathrm{g} / \mathrm{mmol}$ creatinine), serum $\beta-2-$ microglobulin (S- $\beta 2 \mathrm{MG} ; \mathrm{mg} / \mathrm{l}$ ), serum albumin $(\mathrm{g} / \mathrm{l})$, and protein:creatinine ratio in the first and second morning urine (PCR-U1 and PCR-U2) were assessed as described previously. ${ }^{7811}$ A PCR$\mathrm{U} 1>108$ or a PCR-U2 $>127$ indicate abnormal proteinuria. The GAG excretion was expressed as a concentration $(\mathrm{mg} / \mathrm{l})$ standardised for a diuresis of $1 \mathrm{ml} / \mathrm{min}^{8}$

Several confounders were considered because serum laminum may be affected by liver dis- 
eases, ${ }^{1213}$ scleroderma, ${ }^{14}$ cancer, ${ }^{15}$ diabetes, ${ }^{16}$ and perhaps age or glomerular filtration rate. ${ }^{2}$ All workers who had a clinical history with past or present disease capable of affecting the kidney (systemic diseases such as hypertension or diabetes included) or the urinary tract, or who had regularly taken drugs for at least one month before the clinical examination constituted the "renal" disease group. Regular drug consumption was taken into consideration because it can affect sensitive markers of renal disease. Most workers in this group (52; $44 \cdot 1 \%$ ) were hypertensive (systolic blood pressure $\geqslant 160$ or diastolic pressure $\geqslant 95 \mathrm{~mm} \mathrm{Hg}$ ). There were too few workers with renal parenchymal disease to constitute a more specific group.

As Gosling and Beevers ${ }^{17}$ showed that $\mathrm{U}$-alb can already increase above blood pressure values $\geqslant 140$ or $\geqslant 90 \mathrm{~mm} \mathrm{Hg}$, we also defined subgroups according to these cut off values. A "higher" blood pressure (HBP) was then defined as any value $\geqslant 140$ or $\geqslant 90 \mathrm{mmHg}$, a "lower" blood pressure (LBP) as values $<140$ and $<90 \mathrm{~mm} \mathrm{Hg}$. Mean blood pressure is the sum of diastolic blood pressure and one third of the difference between systolic and diastolic blood pressure.

The digestive disease group included all workers with a clinical history of digestive, biliary, pancreatic, or hepatic disease, or regular drug consumption as defined earlier. The liver disease group included only workers with a history of hepatic disease. Most patients $(11 ; 58 \%)$ had a clinical history of hepatitis. Further confounding factors were creatinine clearance, age, alcohol consumption (in drinks $/$ day $^{18}$ ), and exposure to heavy metals. No worker suffered from scleroderma or primary biliary cirrhosis.

Logistic regression analysis, variance analyses, and multiple regression (backward method) were used to compare groups or to study a variable. ${ }^{19} 20$ If necessary, non-parametric tests or logarithmic transformations were used. For exposure score or exposure duration a normalisation or a better residual distribution could only be achieved by taking the square root.

\section{Results}

Age, body weight, body height, smoking (packyears), LED, LES, S- $\beta 2 M G$, S-creatinine, creatinine clearance, PCR-U2, U-alb, GAG, and the prevalences of workers with symptoms of excessive exposure, daily alcohol consumption, liver or "renal" disease, and hypertension were compared between the group with missing values for one or more of the most important biological measures (serum laminin, U-alb, GAG) and the group without missing values. Most missing values $(n=20$; $80 \%$ ) was due to a missing serum laminin determination (too little serum). These comparisons did not show any trend with respect to exposure, renal function, or possible confounders that could have biased the results. Medians (10th-90th percentiles) were 37 years $(21-57)$ for age, 8.9 years $(0-30 \cdot 2)$ for LED, 11.5 arbitrary units (0-42.8) for LES, $1.87 \mathrm{U} / \mathrm{ml}(1.51-2.37)$ for S-lam, $1.70 \mathrm{mg} / \mathrm{l}$ $(1 \cdot 30-2 \cdot 10)$ for $\mathrm{S}-\beta 2 \mathrm{MG}, 91.0 \mu \mathrm{mol} / 1(79 \cdot 0-103 \cdot 0)$ for serum creatinine, $110.4 \mathrm{ml} / \mathrm{min} / 1.73 \mathrm{~m}^{2}$ $(78 \cdot 3-137 \cdot 4)$ for creatinine clearance; 103.3 $(77 \cdot 8-171 \cdot 1)$ for PCR-U2; $5.08 \mu \mathrm{g} / \mathrm{min}$ $(2 \cdot 40-20 \cdot 28)$ for U-alb, and $45 \cdot 6(34 \cdot 5-62 \cdot 5)$ for GAG (concentration standardised for a diuresis of $1 \mathrm{ml} / \mathrm{min}$ ), in the group without missing values.

Concentrations of serum laminin correlate with age $(r=0 \cdot 17, \mathrm{p} \leqslant 0.001)$ and systolic and diastolic blood pressure $(r \geqslant 0 \cdot 14, \mathrm{p} \leqslant 0.005)$. Correlations with serum creatinine, S- $\beta 2 M G$, or creatinine clearance were at best of borderline significance $(p \geqslant 0.07)$. The serum laminin concentrations were not raised in the digestive disease group $(p=0.29)$ and did not show any pattern related to smoking $(p=0 \cdot 22)$ or alcohol consumption $(p=0.32)$, which was less than about $40 \mathrm{~g}$ ethanol/day in $240(90.9 \%)$ workers. A slight but not significant trend towards higher values was found in the liver disease group $(p=0.39)$ whereas the increase was clear in the "renal" disease group $(p=0.01)$ and still more in the hypertensive group or the group with higher blood pressure ( $p \leqslant$ 0.0001 ) although there were few patients with severe hypertension (90th percentile : 158 and 98 $\mathrm{mm} \mathrm{Hg}$, highest values $200 / 100$ and $180 / 120$ for systolic and diastolic blood pressure respectively). Belonging to the liver or "renal" disease group and blood pressure, age, and creatinine clearance were therefore considered as possible determinants in further analyses.

In a first step, the influence of LED or LES, and blood pressure or "renal" disease on serum laminin concentration was examined before taking possible confounders into account. Table 1 shows that the blood pressure increased serum laminin concentration more in the exposed than in the non-exposed workers. The same trend was found in the worker group with long duration of exposure ( $\geqslant 10$ years). Table 1 is designed to allow a direct comparison with our previous results and shows that serum laminin behaved similarly to U-alb or NAG. ${ }^{7}$ The other possible combinations (LES quartiles with the factor "renal" disease, or ED1-3 with LBP or HBP groups) showed a similar trend (details not given). In the multiple regression analysis the interaction term between mean blood pressure and exposure index (LED or LES) was always statistically significant and furthermore, the coefficients remained fairly stable after the introduction of the confounders (table 2). As discussed later, the negative regression coefficient is probably due to a 
Table 1 Occupational hydrocarbon exposure, hypertension, and serum laminin concentration

\begin{tabular}{|c|c|c|c|c|c|c|c|c|c|}
\hline & \multicolumn{4}{|c|}{ Laminin concentration } & & \multicolumn{4}{|c|}{ Laminin concentration } \\
\hline & $\begin{array}{l}\text { No of } \\
\text { workers }\end{array}$ & Median & $\begin{array}{l}\text { 90th } \\
\text { percentile }\end{array}$ & p Value & & $\begin{array}{l}\text { No } \\
\text { of workers }\end{array}$ & Median & $\begin{array}{l}\text { 90th } \\
\text { percentile }\end{array}$ & P Value \\
\hline \multicolumn{10}{|l|}{ LBP } \\
\hline ESQ1 & 50 & 1.87 & $2 \cdot 47$ & & ED1 & 27 & $1 \cdot 89$ & $2 \cdot 83$ & \\
\hline ESQ2 & 48 & 1.79 & $2 \cdot 08$ & & $\mathrm{ED} 2$ & 57 & 1.79 & $2 \cdot 05$ & \\
\hline ESQ3 & 40 & $1 \cdot 81$ & $2 \cdot 26$ & & ED3 & 46 & $1 \cdot 85$ & $2 \cdot 30$ & \\
\hline ESQ4 & 40 & 1.81 & $2 \cdot 37$ & & & & & & \\
\hline \multicolumn{10}{|l|}{$\begin{array}{l}\text { HBP } \\
\text { group }\end{array}$} \\
\hline ESQ1 & 10 & 1.96 & $2 \cdot 34$ & 0.54 & ED1 & 8 & $2 \cdot 18$ & - & $0 \cdot 22$ \\
\hline ESQ2 & 15 & $2 \cdot 13$ & $2 \cdot 47$ & 0.006 & ED2 & 27 & $1 \cdot 78$ & $2 \cdot 33$ & 0.33 \\
\hline ESQ3 & 13 & $2 \cdot 04$ & $2 \cdot 72$ & 0.003 & ED3 & 70 & 1.95 & $2 \cdot 40$ & 0.07 \\
\hline ESQ4 & 24 & 1.99 & $2 \cdot 56$ & $0 \cdot 007$ & & & & & \\
\hline
\end{tabular}

See methods section for definitions of LBP, HBP, "renal" disease group, and ED1-3; ESQ1-4 = exposure score quartiles (from $1=$ lowest to $4=$ highest); $p$ by Mann-Whitney U test for the difference between LBP and HBP workers in each ESQ or between "renal" disease groups in each ED category.

selection effect. Concentrations of serum laminin were not raised in the workers complaining of symptoms of excessive recent exposure. The same calculations were done with the present job as an index of present exposure but some subgroups were too small to allow a meaningful statistical analysis.

We also assessed the two basal membrane components, serum laminin and GAG, as possible predictors of glomerular damage. The markers of glomerular damage used were total proteinuria, erythrocyturia, and $U$-alb. Neither serum laminin nor GAG were clearly associated with PCR values and neither was a significant predictor of abnormal proteinuria (PCR-U1 > 108 or PCR-U2 > 127). GAG but not serum laminin was weakly associated with an increased erythrocyturia ( $>600$ erythrocytes $/ \mathrm{ml})(p=0.05)$. In the multiple regression analysis with GAG excretion and serum laminin concentration, GAG excretion, but not serum laminin concentration, correlated with U-alb (determination coefficient $0 \cdot 13$ ). After considering RBP concentration (as an indicator of proximal tubule dysfunction) and NAG activity (as an indicator of tubular damage), the determination coefficient increased $(0 \cdot 22)$. This effect was inde- pendent of blood pressure, which still increased the determination coefficient $(0.27)$ without removing the three other variables. In particular, GAG remained a good predictor of albuminuria (partial regression coefficient: $0.009, p<0.0001$ ) whereas the serum laminin effect was non-significant in all regressions. The same results were obtained with the fractional albumin clearance, which is thought to be a more reliable indicator of the properties of the glomerular filter than the albumin excretion rate.

It could be argued that the interaction between blood pressure and exposure for serum laminin concentration is questionable because the exposure indicators are based on the occupational history only. Therefore, the regression analyses were done again after substitution of cumulative HA excretion in the LED or the LES. Simultaneously, the exposure effect on the variables (U-alb, NAG, and to a lesser extent PCR or erythrocyturia) that proved interesting in our previous work ${ }^{7}$ were re-examined with this exposure index. Only the printers $(n=25)$ and the controls $(n=48)$ were included in these analyses. Care was taken to exclude six printers who had had an important solvent exposure other than that due to work in the plant where

Table 2 Serum laminin

\begin{tabular}{|c|c|c|c|c|c|c|}
\hline \multirow[b]{2}{*}{ Exposure index } & & \multicolumn{4}{|c|}{ Other independent variables } & \multirow[b]{2}{*}{ Adjusted $r^{2}$} \\
\hline & & $M B P$ & Age & Interaction & "Renal" disease & \\
\hline $\begin{array}{l}\text { LED } \\
(n=240) \\
\text { LES } \\
(n=240) \\
\text { cumulative HA } \\
(n=62)\end{array}$ & $\begin{array}{l}-0.22 \\
(0.0001) \\
-0.18 \\
(0.0003) \\
-0.75 \\
(0.03)\end{array}$ & $\begin{array}{l}\mathbf{R} \\
\mathbf{R} \\
\mathbf{R}\end{array}$ & $\begin{array}{l}0.001 \\
(0.01) \\
0.001 \\
(0.005) \\
\mathrm{R}\end{array}$ & $\begin{array}{l}0 \cdot 11 \\
(0 \cdot 0001) \\
0 \cdot 09 \\
(0 \cdot 0004) \\
0 \cdot 37 \\
(0 \cdot 03)\end{array}$ & $\begin{array}{l}\mathbf{R} \\
\mathbf{R} \\
\mathbf{R}\end{array}$ & $\begin{array}{l}0.09 \\
0.09 \\
0.06\end{array}$ \\
\hline
\end{tabular}

Dependent variable $=$ serum laminin. Each multiple regression analysis considers another exposure indicator but the same other independent variables. The values indicated are partial regression coefficients (B) and levels of significance (in parentheses); $R=$ removed from the backward regression analysis (non-significant); $\mathrm{r}^{2}=$ adjusted determination coefficient; MBP $=$ mean blood pressure. Interaction = interaction between MBP and LED, LES, or cumulative HA, respectively; for renal disease; $1=$ no, $2=$ yes. 
Table 3 Multiple regression analysis with albumin excretion rate as dependent variable

\begin{tabular}{|c|c|c|c|c|c|c|c|}
\hline \multicolumn{8}{|c|}{ Set of independent variables } \\
\hline $\begin{array}{l}G A G \\
\text { excretion }\end{array}$ & $\begin{array}{l}\text { Serum } \\
\text { laminin } \\
\text { concentration }\end{array}$ & $\begin{array}{l}\text { Cumulative } \\
H A\end{array}$ & $M B P$ & Interaction & Renal disease & $\begin{array}{l}R B P \\
\text { concentration }\end{array}$ & $r^{2}$ \\
\hline \multirow{2}{*}{$\begin{array}{l}1.21 \\
(0.008) \\
\mathrm{NC}\end{array}$} & $\mathbf{R}$ & NC & NC & NC & NC & NC & $0 \cdot 10$ \\
\hline & NC & $\begin{array}{l}-4 \cdot 23 \\
(0 \cdot 01)\end{array}$ & $\mathbf{R}$ & $\begin{array}{l}2 \cdot 11 \\
(0.01)\end{array}$ & $\mathbf{R}$ & NC & 0.07 \\
\hline \multirow{2}{*}{$\begin{array}{l}1 \cdot 21 \\
(0 \cdot 008) \\
1 \cdot 20 \\
(0 \cdot 009)\end{array}$} & $\mathbf{R}$ & $\begin{array}{l}-3.75 \\
(0.03)\end{array}$ & $\mathbf{R}$ & $\begin{array}{l}1.88 \\
(0.03)\end{array}$ & $\mathbf{R}$ & NC & $0 \cdot 17$ \\
\hline & $\mathbf{R}$ & $\begin{array}{l}-3.73 \\
(0.03)\end{array}$ & $\mathbf{R}$ & $\begin{array}{l}1 \cdot 87 \\
(0.03)\end{array}$ & $\mathbf{R}$ & $\mathbf{R}$ & $0 \cdot 16$ \\
\hline
\end{tabular}

Each equation was run with a different set of independent variables; the partial regression coefficient (B) and the level of significance (in parentheses) are indicated; NC = none considered in this run; $\mathrm{R}=$ removed from the backward regression analysis (non-significant); $\mathrm{MBP}=$ mean blood pressure; $\mathrm{r}^{2}=$ adjusted coefficient of determination; $\mathrm{n}=66$ (after exclusion of 6 workers; see text).

the biological monitoring was done. The interaction between blood pressure and exposure increased significantly the albumin excretion rate (table 3), the fractional albumin clearance (partial regression coefficient of the interaction: $2 \cdot 18$, $\mathrm{p}=0.01$ ), the serum laminin (table 2), and the NAG activity (partial regression coefficient: $1 \cdot 27$, $p=0.003)$. This interaction effect was also associated with an abnormal PCR-U1 and PCR-U2 $(r=$ 0.25 and $0.20, p=0.01$ and 0.03 respectively; logistic regression analysis). Only the erythrocyturia was not dependent on this interaction.

As the decline in creatinine clearance is age related, the interaction age-cumulative HA excretion was used to examine the interaction effect on this variable. Again the interaction was significant (partial regression coefficient $=-0.60, p=0.01$ ). The results were similar if S- $\beta 2 M G$ was used instead of creatinine clearance.

This interaction effect cannot be easily explained by confounders as the known confounders were considered in all equations and as the inclusion of the six excluded printers (see earlier) did not change the results. Moreover, these results cannot be explained by a high correlation between LED or LES and cumulative HA excretion because this correlation is weak $(r=0 \cdot 16, \mathrm{p}=0 \cdot 25)$. As in the multiple regression analysis with age, body mass index, regular drug taking, cumulative HA excretion, and the factor "renal" disease, only age and body mass index were associated with mean blood pressure, an association between mean blood pressure and cumulative HA excretion cannot be viewed as a confounder. Finally, as heavy metal exposure was more frequent in the control group than in the printers' group $(50.0 \% v 8.1 \%)$ it cannot be made responsible for the effects found.

It should be emphasised that the printers' exposure to toulene was never very high between 1978 and 1987. The median of the individual mean yearly HA excretions for this 10 year period ranged from a maximum of 1.5 in 1984 to a minimum of $0.5 \mathrm{mmol} \mathrm{HA} / \mathrm{mmol}$ creatinine in 1987 . The yearly median excretion diminished (range of medians: $1 \cdot 1-1.5$ between 1978 and $1984,0.5-1.0$ between 1985 and 1987). The highest individual yearly mean was $3.3 \mathrm{mmol} \mathrm{HA} / \mathrm{mmol}$ creatinine and was registered in 1984 (present biological exposure limit: $\quad 1.58 \mathrm{mmol} \mathrm{HA} \mathrm{mmol} / \mathrm{creatinine}$ ). The median cumulative HA excretion was $10.1 \mathrm{mmol}$ $\mathrm{HA} / \mathrm{mmol}$ creatinine (range: $0 \cdot 3-15 \cdot 3$ ). Enough workers remained in the plant between 1978 and 1987 to allow comparison of their yearly HA excretion with the yearly HA excretion of those who left? the printing house. A cross sectional comparison of the yearly HA excretion of the two groups did not show differences between them for any year $(0 \cdot 14$ $\leqslant \mathrm{p} \leqslant 0.99)$ if the comparison was made year by year. An analysis of the cumulative HA excretion over the whole employment time, however, showed that the printers' group was not homogeneous. There was an obvious trend towards a lower exposure intensity in the workers who remained in the plant for a long time $(p=0.0002)$.

\section{Discussion}

The most interesting result from this study was the finding of an interaction between hypertension and cumulated HA excretion in a population of printers exposed to toluene whose urinary HA excretion was regularly monitored. This interaction was significantly associated with an abnormal proteinuria, an increased serum laminin concentration, albumin excretion rate, and NAG activity. It cannot be explained by the confounders studied. Furthermore, exposure to hydrocarbons seemed to accelerate the age dependent decline in creatinine clearance. These results agree well with the conclusions drawn for several markers of kidney damage in a hydrocarbon exposed population in which anamnestic exposure indices (LED, LES) were used instead of biological monitoring. ${ }^{78}$ 
The second interesting result was the relation between U-alb and the two markers of glomerular basal membrane disturbances, serum laminin concentration and GAG excretion. Although both serum laminin concentration and GAG excretion are thought to increase in glomerular damage 21 GAG, but not serum laminin seems to be a predictor of U-alb, probably because its negative charges repel the albumin molecule and limit its filtration. ${ }^{22}$ The hydrocarbon metabolites and hypertension could induce a metabolic disturbance at the level of glomerular basal membranes causing a loss of polyanionic charges and an increased escape of albumin. One possible explanation of these relations might be that hypertensive workers produce more nephrotoxic metabolites in the form of thioethers than normotensive workers. This hypothesis is based, however, only on animal experiments ${ }^{2324}$ and has to be verified in humans.

The third interesting point is the influence of hydrocarbon exposure and hypertension on the serum laminin concentration. In this regard, the serum laminin concentration showed the same pattern as that already described for several other markers of glomerular and tubular damage with LED and LES. ${ }^{78}$ The lack of effect of alcohol and hepatitis is not surprising because the study population included few heavy drinkers and because most of the hepatitis cases were probably of type A. In the same study population, alcohol consumption increased GGT and ALT activities $(p \leqslant 0.0001$ and $p=0.09$ respectively, details not shown) so that the lack of effect of alcohol consumption on serum laminin concentration cannot be explained by inaccurate answers to the question about alcohol consumption. With respect to the exposure indices the negative partial regression coefficient may be explained by a selection effect due to the cross sectional study design. Hypertensive workers have a higher risk of cardiovascular complications and, therefore, are more likely to leave the plant because of disease. Furthermore, as shown by the relation between cumulative HA excretion and exposure duration in the printer group, it seems that the most heavily exposed workers, who should have the greatest risk of kidney damage, tended to leave the plant earlier. Selection because of disease and because of heavy exposure should therefore cause the progressive formation of a subgroup of normotensive and healthy workers with a fairly long exposure duration but a rather low exposure intensity. Consequently, as both the cumulative indices and the number of years during which the selection effect works increase with time, the partial regression coefficient becomes negative after taking into account the interaction between hypertension and exposure.
The fact that the lack of homogeneity of the study population is not apparent at first sight is not surprising if the limitations of these exposure indices in the frame of a cross sectional design are considered. The LED only takes the exposure duration into account and neglects the individual differences of the exposure intensity. The LES and the cumulative HA excretion consider both exposure duration and intensity but are unable to distinguish between some years of heavy exposure and many years of low exposure. Only the examination of the exposure of all workers having ever being hired during a period of several years can disclose these exposure differences. We are aware that the lack of medical data does prevent us from proving this explanation but it should be borne in mind that the results of the different kidney function tests are consistent ${ }^{7811}$ and that all important known confounders have been considered for each test without changing the results. Moreover, these opposite processes might also partly explain the fact that cross sectional studies about the nephrotoxicity of organic solvents have often produced conflicting results.

It could be argued that the study results rely on sensitive biochemical markers that are not relevant for prevention. This objection does not apply, however, to abnormal proteinuria or to increased albuminuria. Indeed, both are associated with the progression of renal disease and an increased prevalence of cardiovascular disease. ${ }^{25-28}$ Furthermore, occupational exposure to hydrocarbons is also associated with glomerulonephritis and chronic renal failure. ${ }^{29} 30$ Owing to the occupational, medical, social, and financial consequences of chronic renal disease the practical importance of good control of occupational hydrocarbon exposure is obvious.

We thank Mr P Wüthrich, Mr R Schütz, and the Swiss National Accident Insurance Fund for their generous financial grant and their administrative support, Mrs $M$ Berode, C Berthod, J Pilliod, and Mr F Rey for the analytical work, Mr P May and M A Boillat for their help in searching the records, and Mrs C Scheibling for typing the manuscript.

Requests for reprints to: Dr P Hotz, Institut für Sozial- und Präventivmedizin; Sumatrastrasse 30; CH-8006 Zürich; Switzerland.

1 Timpl R. Structure and biological activity of basement membrane proteins. Eur F Biochem 1989;180:487-502.

2 Horikoshi S, Koide H. Serum laminin P1 fragment concentration in renal diseases. Clin Chim Acta 1991;196:185-92.

3 Shinkai Y, Karai M, Osawa G, Sato M, Koshikawa S Antimouse laminin antibodies in IgA nephropathy and various glomerular diseases. Nephron 1990;56:285-96.

4 Viau C, Bernard A, Lauwerys R, Buchet, JP, Quaeghebeur L, Cornu ME, et al. A cross-sectional survey of kidney function 
in refinery employees. Am $\mathcal{f}$ Ind Med 1987;11:177-87.

5 Mutti A, Alinovi R, Bergamaschi E, Biagini C, Cavazzini S, Franchini I. et al. Nephropathies and exposure to perchloroethylene in dry-cleaners. Lancet 1992;340:189-93.

6 Gerber LM, Shmukler C, Alderman MH. Differences in urinary albumin excretion rate between normotensive and hypertensive, white and nonwhite subjects. Arch Intern Med 1992;152:373-7.

7 Hotz P, Pilliod J, Bernard A, Berode M, Rey P. Mazzocato C, et al. Hydrocarbon exposure, hypertension and kidney function tests. Int Arch Occup Environ Health 1990;62:501-8.

8 Hotz P, Pilliod J, Berode M, Rey F, Boillat MA Glycosaminoglycans, albuminuria and hydrocarbon exposure. Nephron 1991;58:184-91.

9 Hotz P, Tschopp A, Söderström D, Holtz J, Boillat MA. Gutzwiller F. Smell or taste disturbances, neurological symptoms, and hydrocarbon exposure. Int Arch Occup Environ Health 1992;63:525-30.

10 Droz PO, Berode M, Boillat MA, Lob M. Biological monitoring and health surveillance of rotogravure printing workers exposed to toluene. In: Ho MH. Dillon HK, eds. Biological monitoring of exposure to chemicals. Organic compounds. New York: John Wiley 1987:111-31.

11 Hotz P, Pilliod J, Bernard A, Rey F, Boillat MA. Erythrocytes glomérulaires, albuminurie, glycosaminoglycanes et exposition aux hydrocarbures. Schweiz Med Wochenschr 1990;120:999-1004.

12 van Zanten RAA, van Leeuwen RE, Wilson JHP. Serum procollagen III N-terminal peptide and laminin P1 fragment concentrations in alcoholic liver disease and primary biliary cirrhosis. Clin Chim Acta 1988;177:141-6.

13 Annoni G, Colombo M, Cantaluppi MC, Khlat B, Lampertico $P$, Rojkind $M$. Serum type III procollagen peptide and laminin (Lam-P1) detect alcoholic hepatitis in chronic alcohol abusers. Hepatology 1989;9:693-7.

14 Herrmann K, Schulze E, Heckmann M, Schubert I, Meurer $\mathrm{M}$, Ziegler V, et al. Type III collagen aminopropeptide and laminin PI levels in serum of patients with silicosis-associated and idiopathic systemic scleroderma. $\mathrm{Br} f$ Dermatol 1990;123:1-7.

15 Felden F, Renkes P, Fremont S, Chambre JF, Champigneulle B, Gaucher P, et al. Serum laminin P1 in metastatic colon carcinoma. Clin Chem 1991;37:1795-6.

16 Högemann B, Voss B. Altenwerth FJ, Schneider M Rauterberg J, Gerlach U. Concentrations of 7S collagen and laminin P1 in sera of patients with diabetes mellitus. Klin Wochenschr 1986:64:382-5.

17 Gosling P, Beevers DG. Urinary albumin excretion and blood pressure in the general population. Clin Sci 1989;76:39-42.

18 Rollason JG, Pincherle G, Robinson D. Serum gamma glutamyl transpeptidase in relation to alcohol consumption. Clin Chim Acta 1972;39:75-80.

19 SUGI SAS User's Group International. SUGI supplemental library user's guide. 5th ed. Cary, NC: SAS Institute Inc. 1986:1-662.

20 SPSS'X. SPSS-XTM user's Guide. 3rd ed. Chicago: SPSS Inc. 1988:1-1072.

21 Baggio B, Gambaro G, Briani C. Favaro S, Borsatti A. Urinary excretion of glycosaminoglycans and brush border and lysosomal enzymes as markers of glomerular and tubular involvement in kidney diseases. Contrib Nephrol 1984;42:107-10.

22 Reddi AS, Ramamurthi R, Miller M, Dhuper S, Lasker N. Enalapril improves albuminuria by preventing glomerular loss of heparan sulfate in diabetic rats. Biochem Med Metab Biol 1991;45:119-31.

23 Décarie S, Chakrabarti S. Metabolism and hepatorenal toxicity due to repeated exposure to styrene in spontaneously hypertensive rats (SHR). F Toxicol Environ Health 1989; 27:455-65.

24 Craan AG, Malick MA. Structure-nephrotoxicity relationships of glutathione pathway intermediates derived from organic solvents. Toxicology 1989;56:47-61.

25 Yudkin JS, Forrest RD, Jackson CA. Microalbuminuria as predictor of vascular disease in non-diabetic subjects. Lancet 1988;ii:530-3.

26 Ravid M, Savin H, Lang R, Jutrin I, Shoshana L, Lishner M. Proteinuria, renal impairment, metabolic control, and blood pressure in type 2 diabetes mellitus. A 14-year follow-up report on 195 patients. Arch Intern Med 1992;152:1225-9.

27 Messent JWC, Elliott TG, Hill RD, Jarrett RJ, Keen $H_{\text {, }}$ Viberti GC. Prognostic significance of microalbuminuria in insulin-dependent diabetes mellitus: a twenty-three year follow-up study. Kidney Int 1992;41:836-9.

28 Kannel WB, Stampfer MJ, Castelli WP, Verter J. The prognostic significance of proteinuria: The Framingham study. Am Heart f 1984;108: 1347-52.

29 Bell GM, Gordon ACH, Lee P, Doig A, MacDonald MK, Thomson $\mathrm{D}$, et al. Proliferative glomerulonephritis and exposure to organic solvents. Nephron 1985;40:161-5.

30 Steenland NK, Thun MJ, Fergusson CW, Port FK. Occupational and other exposures associated with male end-stage renal disease: A case/control study. Am $\mathcal{F}$ Public Health 1990;80:153-9.

Accepted 8 February 1993 